\title{
High-Order Harmonic Generation Yielding Tunable Extreme-Ultraviolet Radiation of High Spectral Purity
}

\author{
F. Brandi, ${ }^{*}$ D. Neshev, ${ }^{\dagger}$ and W. Ubachs \\ Laser Centre, Department of Physics and Astronomy, Vrije Universiteit ${ }^{\ddagger}$, De Boelelaan 1081, 1081 HV Amsterdam, The Netherlands
}

(Received 3 March 2003; published 14 October 2003)

\begin{abstract}
Production of extreme-ultraviolet radiation by high-order harmonic generation is demonstrated to yield unprecedented spectral purity of $\lambda / \Delta \lambda=2.5 \times 10^{5}$ at wavelengths covering the entire range $40-$ $100 \mathrm{~nm}$. Tunability and sub- $\mathrm{cm}^{-1}$ bandwidth of the harmonics are demonstrated in recordings of the $\mathrm{He}$ $(1 s 4 p)$ and $\operatorname{Ar}\left(3 p^{5} 3 d^{\prime}\right)$ resonance lines at 52.2 and $86.6 \mathrm{~nm}$. Frequency shift of the harmonics due to chirp-induced phenomena are investigated and found to be small, resulting in a frequency accuracy of about $5 \times 10^{-7}$ in the domain of extreme-ultraviolet radiation.
\end{abstract}

DOI: 10.1103/PhysRevLett.91.163901

PACS numbers: 42.65.Ky, 32.30.Jc, 42.72.Bj, 42.79.Nv

A key feature of the nonlinear optics in the nonperturbative regime, i.e., at field intensities above $10^{13} \mathrm{~W} / \mathrm{cm}^{2}$, is the generation of a plateau of nearly equally intense harmonics of odd order extending to a certain cutoff point. An explanation for these experimental features is provided by the semiclassical and intuitive recollision model in which the high field response is considered in three consecutive steps [1]: first the atom is ionized, then the expelled electron accelerates in the laser field, and finally it recombines with the parent ion radiating one harmonic photon. The universal cutoff in high-order harmonic generation (HHG) processes exists at $I_{p}+3.17 U_{p}$, with $I_{p}$ the ionization potential of the atoms and $U_{p}=$ $e^{2} E^{2} / 4 m_{e} \omega_{0}^{2}$ the mean kinetic (ponderomotive) energy of a quasifree electron quivering in the laser field in the neighborhood of the ionic core. Alternatively, nonperturbative calculations with the assumption of single-atom response (macroscopic effects ignored) confirmed results on the plateau and cutoff [2]. The characteristic features were found in early experimental studies, where laser pulses of tens of ps were used [3], as well as in studies where fs pulses were used, giving rise to higher order harmonics (see, e.g., Ref. [4]). Current activities in HHG research aim at the production of coherent shortwavelength radiation with special characteristics that cannot be achieved by alternative sources. At the one end is the strive for attosecond pulses [5]; at the other is the production of coherent light in the extreme-ultraviolet (XUV) domain, down to $x$ rays in the water window [6]. The use of hollow-core fibers was shown to induce strongly enhanced harmonic yields at specific orders [7] and at the same time a greatly improved spatial coherence of the XUV output [8].

For frequency-domain spectroscopic applications the temporal coherence or spectral bandwidth of the radiation is of crucial importance. In the regime of low-order harmonics (in the perturbative regime at field intensities below $10^{12} \mathrm{~W} / \mathrm{cm}^{2}$ ) coherent XUV radiation with a spectral purity of $\lambda / \Delta \lambda>10^{7}$ has been demonstrated, with the use of Fourier-transform (FT) limited nanosecond pulsed lasers, for the 5th harmonic [9] and for resonance-enhanced wave mixing [10]. In contrast to these studies, in the nonperturbative regime of HHG the focus has sparsely been on achieving continuous tunability of the output radiation or on enhancing the spectral purity. A noteworthy exception is a picosecond setup at Lund, producing coherent and continuously tunable XUV radiation in the range $35-100 \mathrm{~nm}$ at a spectral purity of $\lambda / \Delta \lambda \sim 10^{4}$ [11]. That source was used to perform twocolor pump-probe experiments on the $1 s 2 p$ and $1 s 3 p$ transitions in $\mathrm{He}$ at 58 and $54 \mathrm{~nm}$, revealing accurate values of lifetimes and absolute photoionization cross sections [12]. As an alternative to laser-based harmonic sources, some state-of-the-art third generation synchrotron beam lines, equipped with coherence improving undulators and high-resolution monochromators, achieve spectral purities of $\lambda / \Delta \lambda \sim 10^{5}[13,14]$.

In this Letter we extend for the first time the HHG regime to pulses of $300 \mathrm{ps}$ duration, and demonstrate experimentally plateau harmonics with unprecedented bandwidth, resulting in a spectral purity of $\lambda / \Delta \lambda \sim$ $2.5 \times 10^{5}$. The high degree of coherence is combined with continuous tunability and full coverage of the wavelength range $40-100 \mathrm{~nm}$. The basic concept lies in the use of well-characterized, energetic, FT-limited, wavelength-tunable laser pulses of 300 ps duration for harmonic conversion on the plateau. Such laser pulses are established in a novel design optical system, where compression via stimulated Brillouin scattering is responsible for providing the $300 \mathrm{ps}$ pulses, rather than mode locking. The setup is displayed in Fig. 1. The core of the laser system, described in detail in Ref. [15], is a three-stage traveling-wave pulsed-dye amplifier (PDA) transforming fixed-wavelength pulses at $532 \mathrm{~nm}$ into FT-limited tunable pulses (bandwidth $\sim 1.5 \mathrm{GHz}$ ) in the near-infrared wavelength range. The PDA is seeded with the light of a 


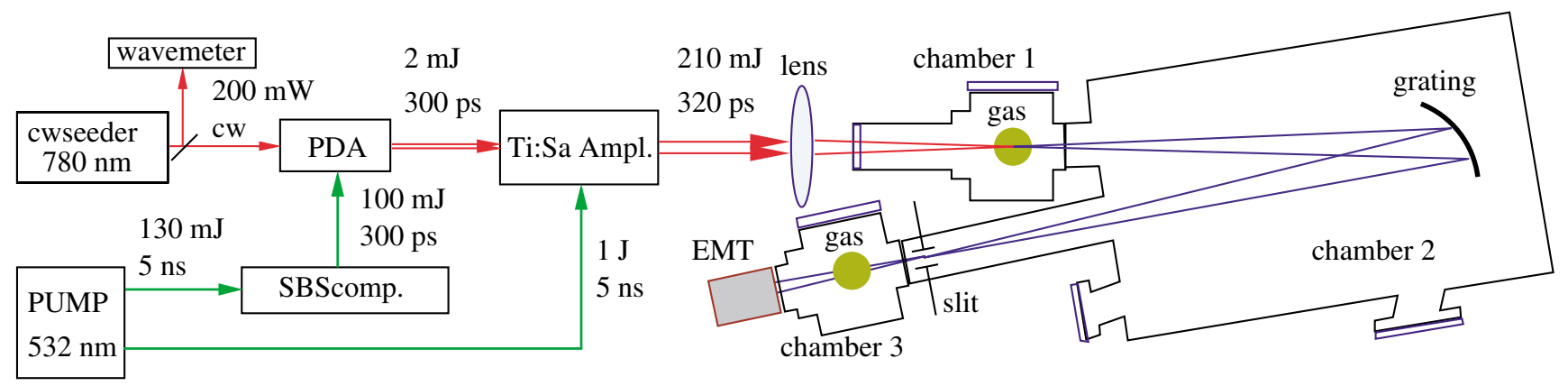

FIG. 1 (color online). Schematic of the experimental setup. The vacuum chambers are differentially pumped, and have a common background pressure of $2 \times 10^{-7}$ mbar.

continuous wave (cw) Ti:sapphire ring laser and its output is further amplified in Ti:sapphire amplifiers. Since the frequency of the seed laser determines the frequencies further on in the optical system, its advantageous wavelength tunability performance holds for the entire setup and, via the harmonics, for the generated XUV output. Pulses of $210 \mathrm{~mJ}$ with $320 \mathrm{ps}$ duration and $10 \mathrm{~Hz}$ repetition rate are produced. The performances of the laser system are demonstrated around $780 \mathrm{~nm}$ using LDS 765 dye in the PDA, but its operation can be extended to the entire Ti:sapphire range $(700-970 \mathrm{~nm})$.

High-order harmonics are generated in a gas jet (Ar and $\mathrm{Kr}$ are used) produced with a piezoelectric valve mounted on a translational stage in a vacuum chamber. The fundamental laser pulses are focused in the jet with a $20 \mathrm{~cm}$ focal length lens to a spot of $40 \mu \mathrm{m}$ in diameter, corresponding to a peak intensity of $5 \times 10^{13} \mathrm{~W} / \mathrm{cm}^{2}$. The harmonics are separated by a spherical normal incidence Au-coated grating with $1 \mathrm{~m}$ radius of curvature (Spectrogon, 1200 grooves $/ \mathrm{mm}$ ). The grating is used in a constant-deviation configuration (deviation angle $=11^{\circ}$ ) and the harmonics are selected with a $1 \mathrm{~mm}$ slit. The intensity of the XUV radiation is measured with an electron multiplier tube (EMT) (Electron Tubes Model 642/2EMB).

In Fig. 2(a) the harmonic spectrum for Ar, with 3 bars backing pressure and the laser focused at $D=1.5 \mathrm{~mm}$ below the valve orifice is shown. The absolute number of harmonic photons generated per pulse, reported in Fig. 2(b), is evaluated using the theoretical grating efficiency, the EMT first dynode ( $\mathrm{BeCu}$ ) quantum efficiency, and the detector gain as specified by the manufacturers. The data of Fig. 2(b) must be considered with some caution since the reflectivity of the gold-coated grating as well as the quantum efficiency of the detector drop sharply at $40 \mathrm{~nm}$. Nevertheless, it may be concluded that the plateau of the harmonics followed by a sudden cutoff, the key feature of HHG, is reproduced in the present study employing much longer pulses than previously used.

The harmonic photon yield is about the same as in the HHG studies employing pulses of about an order of magnitude shorter duration but the same peak intensity
[11,16]. Actually, higher photon yield is expected since the peak intensity is sustained for an extended period. However, multiphoton ionization can greatly reduce the neutral atom density during the 300 ps long pulse, thus reducing the harmonic conversion efficiency. To evaluate the degree of ionization, measurements of the photoelectron yield as a function of the laser intensities are performed. The photoelectrons are collected using a grid with positive potential placed under the gas jet, while the laser intensity is controlled using a half-wave plate in combination with a thin-film polarizer. Preliminary measurements in argon indicate an increase of the photoelectron yield for intensities up to $I \sim 3 \times 10^{13} \mathrm{~W} / \mathrm{cm}^{2}$, as shown in the inset of Fig. 2(b). A fit on these data

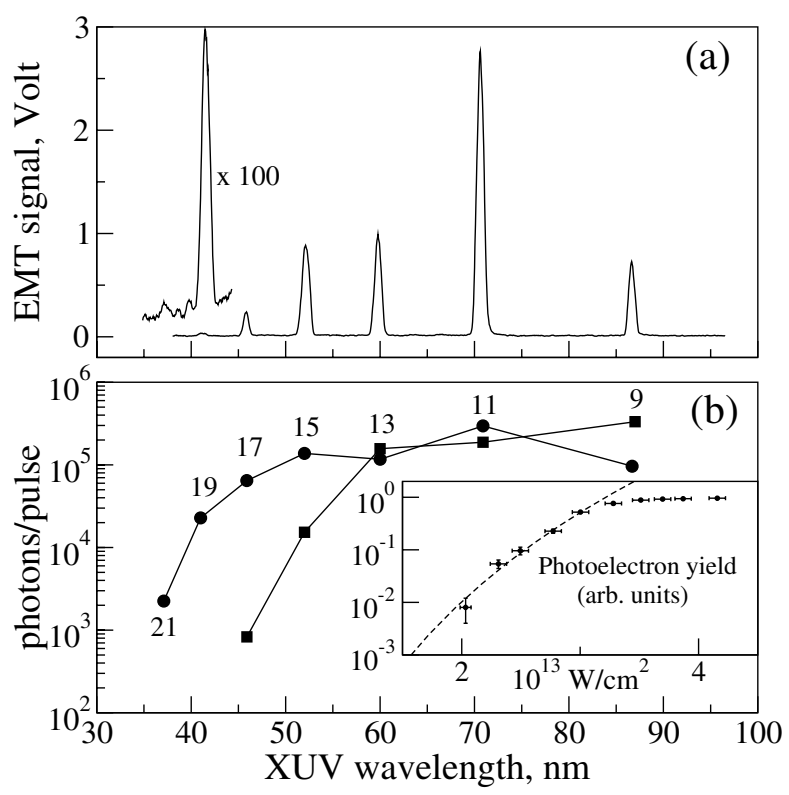

FIG. 2. High-order harmonic generation: spectrum of the harmonics generated in argon (a); absolute harmonic yield in argon (circles) and krypton (squares) (b). The inset in (b) shows the photoelectron yield in argon as a function of the laser intensity: the points are the experimental data, while the dashed line is the result from a weighted power-law fit performed on the lowest data points. 
points indicates a power-law dependence, i.e., $\propto I^{n}$, with $n_{\text {expt }}=9.6(0.5)$, which agrees with the value $n_{\text {theo }}=10$ expected for multiphoton ionization [ten photons at $780 \mathrm{~nm}$ are necessary to ionize argon, $I_{p}(\mathrm{Ar})=$ $15.76 \mathrm{eV}]$. At higher intensities, $I>3 \times 10^{13} \mathrm{~W} / \mathrm{cm}^{2}$, saturation in the photoelectron yield is found. The high degree of ionization on a time scale within the duration of the laser pulse limits the duration of the harmonically produced XUV pulse and hence its photon yield. This cut in the time domain would cause a broadening in the frequency domain beyond the expected bandwidth for a harmonic of a FT-limited pulse, but experimentally this additional broadening is found to be small (see below).

To investigate the frequency-domain characteristics of the high-order harmonics, and also to demonstrate their applicability and continuous tunability, absorption spectroscopy is performed on known narrow atomic transitions. Using these as a ruler, the absolute frequency and the bandwidth of the XUV radiation can be deduced. Absorption is performed in a differentially pumped vacuum chamber using a pulsed jet expansion, set perpendicular to the XUV beam in order to minimize Doppler effects. The density in the absorbing gas jet was kept such that 20\%-40\% peak absorption was measured. Two atomic transitions with extremely well known transition frequencies are used: $\left(3 p^{6}{ }^{1} S_{0} \rightarrow 3 p^{5} 3 d^{\prime}[3 / 2]_{1}\right)$ in $\mathrm{Ar}$ at $86.7 \mathrm{~nm}$ to probe the 9th harmonic generated in $\mathrm{Kr}$ $\left(\lambda_{\text {fund }}=780 \mathrm{~nm}\right)$, and $\left(1 s^{2}{ }^{1} S_{0} \rightarrow 1 s^{1} 4 p^{1} P_{1}\right)$ in $\mathrm{He}$ at $52.2 \mathrm{~nm}$ to probe the 15 th harmonic generated in $\mathrm{Ar}$ $\left(\lambda_{\text {fund }}=783 \mathrm{~nm}\right)$. The frequencies of the resonance lines are taken at $3458611.6(1.5) \mathrm{GHz}$ for $\left(3 p^{6}{ }^{1} S_{0} \rightarrow\right.$ $\left.3 p^{5} 3 d^{\prime}[3 / 2]_{1}\right)$ in argon [17] and 5740807.0(1) GHz for $\left(1 s^{2}{ }^{1} S_{0} \rightarrow 1 s^{1} 4 p^{1} P_{1}\right)$ in helium [18]. The level energies of argon reported by Minnhagen [17] have been confirmed by accurate laser spectroscopy for the $\left(3 p^{6}{ }^{1} S_{0} \rightarrow\right.$ $3 p^{5} 4 s^{\prime}[1 / 2]_{1}$ ) transition at $105 \mathrm{~nm}$ [19]. The level energies of several excited states of helium calculated by Drake and Martin [18] are in agreement with experimental values within $100 \mathrm{MHz}$ [9]. The present investigation is the first laser-based and sub-Doppler absorption measurement on the $4 p$ resonance line of He.

Absolute frequency of the harmonics. - Absorption spectra in the XUV are recorded by scanning the cw ring Ti:sapphire laser, whose frequency $\nu$ is online monitored on a calibrated wave meter (Atos, Lambdameter) with an absolute accuracy of $100 \mathrm{MHz}$. In principle the relation $\nu_{q}=q \times \nu$ gives the frequency of the $q$ th harmonic $\nu_{q}$. In Fig. 3 the absorption spectra of the argon and helium lines are shown for two valve-focus distances, $D=5$ and $1.5 \mathrm{~mm}$, which correspond to different gas densities. The narrowest harmonics spectra are observed at $D=5 \mathrm{~mm}$, full width at half maximum 15 and $30 \mathrm{GHz}$ for the 9th and 15th harmonics, respectively. At first inspection the observed absorption resonances match the predicted frequencies extremely well, indicating that nearly exact harmonics are generated. However, the

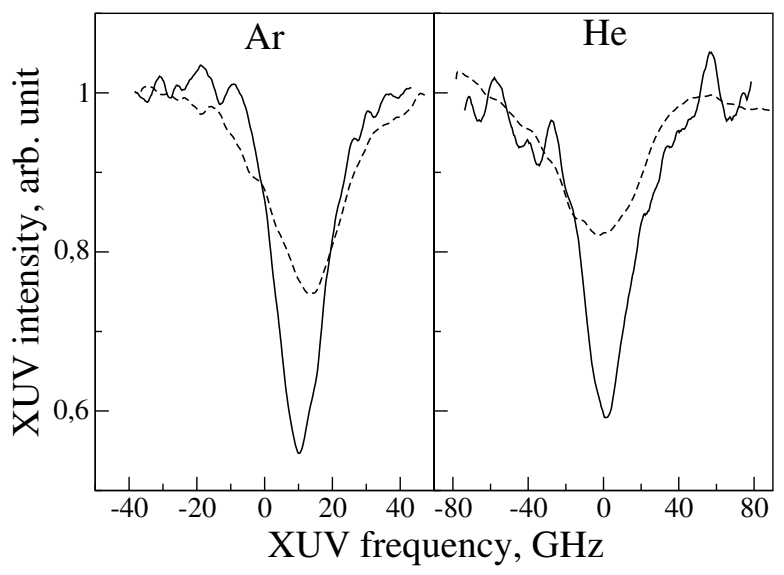

FIG. 3. High-order harmonic spectra: on the left the spectrum of the $\left(3 p^{6} S_{0} \rightarrow 3 p^{5} 3 d^{\prime}[3 / 2]_{1}\right)$ argon transition at $86.7 \mathrm{~nm}$ measured with the 9th harmonic generated in krypton; on the right the $\left(1 s^{2} S_{0} \rightarrow 1 s^{1} 4 p^{1} P_{1}\right)$ helium transition at $52.2 \mathrm{~nm}$ measured with the 15th harmonic generated in argon. The dashed and solid lines correspond to measurements $D=1.5$ and $5 \mathrm{~mm}$, respectively. Zero points on the frequency axes are $3458611.6(1.5) \mathrm{GHz}$ for Ar and $5740807.0(1) \mathrm{GHz}$ for $\mathrm{He}$.

small shifts from center frequency are reproducible and significant, due to the accurate metrology performed. The central frequency of the argon transition is blueshifted by $10 \mathrm{GHz}$, while for the helium transition no shift is observed. The spectra obtained with $D=1.5 \mathrm{~mm}$ (higher gas density in harmonic conversion zone) show broadening, a few $\mathrm{GHz}$ additional blueshift for the argon transition, and a few $\mathrm{GHz}$ redshift for the helium transition. The observed shifts are attributed to frequency chirp, as a result of self-phase modulation of the fundamental radiation due to dynamic nonlinear susceptibility and ionization of the medium, as well as other effects inherent to the HHG process (see, e.g., [20]); shifts of $>1 \%$ are typical with fs pulses at intensities of $10^{15} \mathrm{~W} / \mathrm{cm}^{2}$ [4]. In the present experiment, where longer pulses and lower intensities are employed, the chirp effect is extremely small, allowing one to use HHG for precision spectroscopy in the XUV domain.

If the chirp-induced shift effects are monitored, the absolute uncertainty in the XUV frequency calibration can, after correction, be brought to the $\mathrm{GHz}$ level. Frequency chirp in the amplification chain of the nearinfrared laser system, which is measured at $80 \mathrm{MHz}$ in the fundamental [15] and causing an additional shift of up to $1 \mathrm{GHz}$ in the harmonic, should also be accounted for. Incorporating these frequency shifts and correcting for them, by measuring reference standards as in the present example, will allow for future spectroscopic applications employing high-order harmonics at an absolute accuracy of a few $\mathrm{GHz}$, corresponding to a relative accuracy of about $5 \times 10^{-7}$.

Bandwidths of the harmonics. - The observed widths in the recording of spectral lines, $\Delta \nu_{9, \mathrm{obs}}=15 \mathrm{GHz}$ for 
$3 d^{\prime}$ in $\mathrm{Ar}$ and $\Delta \nu_{15, \mathrm{obs}}=30 \mathrm{GHz}$ for $4 p$ in $\mathrm{He}$, can be used to estimate the bandwidth of the harmonics. Although absorption is recorded in a free jet, the Doppler width is reduced, but not to a negligible extent. Residual Doppler broadenings of $3 \mathrm{GHz}$ for argon and $15 \mathrm{GHz}$ for helium are estimated for an effusive thermal atomic beam with $40^{\circ}$ opening angle, which is typical for a General Valve Series-9 pulsed solenoid valve. The effect of the XUV beam divergence is negligible. Deconvolution of residual Doppler width yields a bandwidth of $\Delta \nu_{9}=$ $13 \mathrm{GHz}$ for the 9th and $\Delta \nu_{15}=20 \mathrm{GHz}$ for the $15 \mathrm{th}$ harmonic. In both cases this corresponds to a resolving power of $\lambda / \Delta \lambda=2.5 \times 10^{5}$ and the bandwidth is well below the $\mathrm{cm}^{-1}$ level. The expected width for the harmonics based on measurements of the width of the fundamental $\Delta \nu$ can be estimated under the assumption of a Gaussian frequency profile $\left(\Delta \nu_{q}=\sqrt{q} \times \Delta \nu\right)$, which was measured for the frequency profile of the fundamental [15] with a width $\Delta \nu \sim 1.5 \mathrm{GHz}$; from this we estimate for the 9th-15th harmonics a bandwidth of $6-10 \mathrm{GHz}$. This is a factor of 2 lower than actually observed. The additional broadening of the harmonics is attributed to frequency chirp effects.

Tunability of the harmonics. - Experiments are demonstrated here for fundamental wavelengths at 780$783 \mathrm{~nm}$, but in principle the entire Ti:sapphire tuning region of 700-970 $\mathrm{nm}$ can be used. In fact only the range $735-950 \mathrm{~nm}$ is necessary for full coverage over the 40-100 XUV region, while using successive harmonics. In addition we have experimented with the use of even harmonics by implementing a frequency-doubling stage before the harmonic conversion in a jet. By this means we successfully produced 8th (via a wave-mixing scheme involving both fundamental and frequencydoubled output) and 10th harmonics, albeit at a slightly lower intensity.

In conclusion, narrow band continuously tunable XUV laser radiation, based on $\mathrm{HHG}$, is demonstrated. An unprecedented spectral purity $\lambda / \Delta \lambda$ of $2.5 \times 10^{5}$ covers the entire $40-100 \mathrm{~nm}$ region with continuous tunability. The HHG conversion efficiency of the present source is small when compared with efficiencies of $10^{-5}-10^{-7}$ achieved using fs pulses and phase matching in gasfilled hollow fibers [7,8]. The use of hollow fiber to improve the harmonic yield will be considered in the future for the present setup. Chirp shift effects in HHG with 300 ps FT-limited pulses are quantified, and it is shown that a frequency accuracy of $5 \times 10^{-7}$ is achieved in the XUV domain. This brings HHG into the realm of high-resolution XUV spectroscopy.

The authors thank the Netherlands Foundation for Fundamental Research of Matter (FOM) for financial support. D. N. acknowledges support via a Marie Curie individual fellowship from the European Commission (Contract No. HPMFCT-2000-00455).

*Email address: brandi@nat.vu.nl

${ }^{\dagger}$ Present address: Nonlinear Physics Group, Australian National University, Canberra.

Electronic address: http://www.nat.vu.nl/ atom

[1] P. B. Corkum, Phys. Rev. Lett. 71, 1994 (1993).

[2] J. L. Krause, K. J. Schafer, and K. C. Kulander, Phys. Rev. A 45, 4998 (1992); Phys. Rev. Lett. 68, 3535 (1992).

[3] A. LHuillier, L. A. Lompré, G. Mainfray, and C. Manus, J. Phys. B 16, 1363 (1983).

[4] K. Miyazaki and H. Takada, Phys. Rev. A 52, 3007 (1995).

[5] M. Drescher, M. Hentschel, R. Kienberg, G. Tempea, C. Spielmann, G. A. Reider, P. B. Corkum, and F. Krausz, Science 291, 1923 (2001).

[6] C. Spielmann, N. H. Burnett, S. Sartania, R. Koppitsch, M. Schnurer, C. Kan, M. Lenzner, P. Wobrauschek, and F. Krausz, Science 278, 661 (1997).

[7] A. Rundquist, C. G. Durfee III, Z. Chang, C. Herne, S. Backus, M. M. Murnane, and H. C. Kapteyn, Science 280, 1412 (1998).

[8] R. A. Bartels, A. Paul, H. Green, H. C. Kapteyn, M. M. Murnane, S. Backus, I. P. Christov, Y. Liu, D. Attwood, and C. Jacobsen, Science 297, 376 (2002).

[9] K. S. E. Eikema, W. Ubachs, W. Vassen, and W. Hogervorst, Phys. Rev. A 55, 1866 (1997).

[10] U. Hollenstein, H. Palm, and F. Merkt, Rev. Sci. Instrum. 71, 4023 (2000).

[11] C. Lyngå, F. Ossler, T. Metz, and J. Larsson, Appl. Phys. B 72, 913 (2001).

[12] A. Johansson, M. K. Raarup, Z.S. Li, V. Lokhnygin, D. Descamps, C. Lyngå, E. Mevel, J. Larsson, C.-G. Wahlström, S. Aloise, M. Gisselbrecht, M. Meyer, and A. LHuillier, Eur. Phys. J. D 22, 3 (2003).

[13] L. Nahon, C Alcazar, J.-L. Marlats, B. Lagarde, F. Polack, R. Thissen, D. Lepere, and K. Ito, Rev. Sci. Instrum. 72, 1320 (2001).

[14] P. Heimann, M. Koike, C.W. Hsu, D. Blank, X. M. Yang, A. G. Suits, Y. T. Lee, M. Evans, C. Y. Ng, C. Flaim, and H. A. Padmore, Rev. Sci. Instrum. 68, 1945 (1997).

[15] F. Brandi, I. Velchev, D. Neshev, W. Hogervorst, and W. Ubachs, Rev. Sci. Instrum. 74, 32 (2003).

[16] X. F. Li, A. LHuillier, M. Ferray, L. A. Lompré, and G. Mainfray, Phys. Rev. A 39, 5751 (1989).

[17] L. Minnhagen, J. Opt. Soc. Am. 63, 1185 (1973).

[18] G.W. F. Drake and W. C. Martin, Can. J. Phys. 76, 679 (1998).

[19] I. Velchev, W. Hogervorst, and W. Ubachs, J. Phys. B 17, L511 (1999).

[20] H. T. Kim, D. G. Lee, K.-H. Hong, J.-H. Kim, I.W. Choi, and C. H. Nam, Phys. Rev. A 67, 051801(R) (2003), and references therein. 